\title{
ON THE NULL-SPACES OF FIRST-ORDER ELLIPTIC PARTIAL DIFFERENTIAL OPERATORS IN $R^{n}$
}

\author{
HOMER F. WALKER ${ }^{1}$
}

Abstract. The objects to be studied are the null-spaces of linear first-order elliptic partial differential operators with domain $H_{1}\left(R^{n} ; C^{k}\right)$ in $L_{2}\left(R^{n} ; C^{k}\right)$, the first-order coefficients of which become constant and the zero-order coefficients of which vanish outside a compact set in $\boldsymbol{R}^{n}$. An example is given of an operator of this type which has a nontrivial null-space. It is shown that the dimension of the null-space of such an operator is finite for any number $n$ of independent variables, and that this dimension is an upper-semicontinuous function of the operator in a certain sense.

1. Introduction. As usual, let $L_{2}\left(\boldsymbol{R}^{n} ; \boldsymbol{C}^{k}\right)$ denote the Hilbert space of equivalence classes of $C^{k}$-valued functions on $R^{n}$ whose absolute values are Lebesgue-square-integrable over $R^{n}$. Let $H_{1}\left(R^{n} ; C^{k}\right)$ denote the Hilbert space consisting of those elements of $L_{2}\left(R^{n} ; C^{k}\right)$ which have (strong) first partial derivatives in $L_{2}\left(R^{n} ; C^{k}\right)$. Denote the usual norms on $L_{2}\left(R^{n} ; C^{k}\right)$ and $H_{1}\left(R^{n} ; C^{k}\right)$ by \|\| and \|\|$_{1}$, respectively. Consider a linear first-order partial differential operator

$$
A_{0} u(x)=\sum_{i=1}^{n} A_{i} \frac{\partial}{\partial x_{i}} u(x)
$$

with domain $H_{1}\left(R^{n} ; C^{k}\right)$ which has constant coefficients and no zeroorder term. Suppose $A_{0}$ is elliptic in the sense that

$$
\operatorname{det}\left|\sum_{i=1}^{n} A_{i} \xi_{i}\right| \neq 0
$$

for all nonzero $\xi$ in $R^{n}$. It is easily seen via Fourier transforms that the only square-integrable solution of the equation $A_{0} u=0$ is the trivial one; in other words, the null-space $N\left(A_{0}\right)$ of $A_{0}$ is $\{0\}$. The objective of this paper is to shed some light on the nature of the null-spaces of operators obtained by allowing the coefficients of such an operator $A_{0}$

Presented to the Society, January 21, 1971; received by the editors December 22, 1970.

AMS 1969 subject classifications. Primary 3513, 3580, 3544; Secondary 3530, 4765.

Key words and phrases. Null-spaces of first-order elliptic operators, perturbation of first-order elliptic operators.

1 Most of the results in this paper are contained in the author's doctoral dissertation written at New York University. The author wishes to express gratitude to Professor Peter D. Lax for his guidance in the preparation of this material. 
to vary in a certain way inside a ball of finite radius about the origin in $R^{n}$.

2. Description of the problem and summary of results. Given a positive $R$ and an operator $A_{0}$ of the type described above, let $E\left(A_{0}, R\right)$ denote the set of linear first-order partial differential operators $A$ of the form

$$
A u(x)=\sum_{i=1}^{n} A_{i}(x) \frac{\partial}{\partial x_{i}} u(x)+B(x) u(x)
$$

with domain $H_{1}\left(\boldsymbol{R}^{n} ; C^{k}\right)$, the $k \times k$ coefficient matrices of which are such that the following conditions are satisfied:

(i) The matrices $A_{i}(x)$ and $B(x)$ are continuous complex-valued functions of $x$ on $R^{n}$, and the functions $A_{i}(x)$ have continuous first derivatives.

(ii) The operator $A$ is elliptic, i.e.,

$$
\operatorname{det}\left|\sum_{i=1}^{n} A_{i}(x) \xi_{i}\right| \neq 0
$$

for all $x$ and all nonzero $\xi$ in $R^{n}$.

(iii) The coefficients of $A$ are equal to the coefficients of $A_{0}$ for $|x| \geqq R$.

The operators in $E\left(A_{0}, R\right)$ are those elliptic operators obtained by adding a "perturbing" operator to $A_{0}$ whose coefficients vanish outside the set $\{x:|x| \leqq R\}$. The operator $A_{0}$ will be referred to as the unperturbed operator of the set $E\left(A_{0}, R\right)$. If $A$ is an operator in $E\left(A_{0}, R\right)$, then there exist positive constants $c_{1}$ and $c_{2}$ depending on $A$ such that the following standard elliptic estimate

$$
\|u\|_{1} \leqq c_{1}\|u\|+c_{2}\|A u\|
$$

holds for all $u$ in $H_{1}\left(R^{n} ; C^{k}\right)$ [4]. From this estimate, it follows that $H_{1}\left(\boldsymbol{R}^{n} ; \mathbf{C}^{k}\right)$ is a natural domain for such an operator in the sense that the operator is closed on $H_{1}\left(\boldsymbol{R}^{n} ; \boldsymbol{C}^{k}\right)$ and its adjoint operator also has domain $H_{1}\left(\boldsymbol{R}^{n} ; \boldsymbol{C}^{k}\right)$.

The objects to be studied here are the null-spaces $N(A)$ of operators $A$ in $E\left(A_{0}, R\right)$. In the case of one independent variable, the equation $A u=0$ can be integrated to yield an exponential solution when $A$ is in $E\left(A_{0}, R\right)$. Then only the trivial solution can be square-integrable in absolute value over $R^{1}$, and so this case merits no attention. When the number of independent variables is at least two, however, there do exist operators of the type under consideration which have non- 
trivial null-spaces. The construction of one such operator will be carried out here. The main results to be derived in the sequel may be summarized by saying that there are certain estimates, valid when the number of independent variables is greater than or equal to two, which imply that the dimension of the null-space of an operator in $E\left(A_{0}, R\right)$ is finite and depends upper-semicontinuously on the operator in a certain sense.

3. An operator with a nontrivial null-space. Consider the CauchyRiemann operator

$$
A_{0} U\left(x_{1}, x_{2}\right)=\left(\begin{array}{rr}
-1 & 0 \\
0 & 1
\end{array}\right) \frac{\partial}{\partial x_{1}} U\left(x_{1}, x_{2}\right)+\left(\begin{array}{ll}
0 & 1 \\
1 & 0
\end{array}\right) \frac{\partial}{\partial x_{2}} U\left(x_{1}, x_{2}\right)
$$

acting on functions $U$ in $H_{1}\left(R^{2} ; C^{2}\right)$. The function

$$
U_{0}\left(x_{1}, x_{2}\right)=\left(\begin{array}{l}
\operatorname{Re} \frac{1}{\left(x_{1}+i x_{2}\right)^{2}} \\
\operatorname{Im} \frac{1}{\left(x_{1}+i x_{2}\right)^{2}}
\end{array}\right)
$$

defined for $x_{1}^{2}+x_{2}^{2}>0$ satisfies $A_{0} U_{0}\left(x_{1}, x_{2}\right)=0$ for $x_{1}^{2}+x_{2}^{2}>0$ and, moreover, is such that $\left|U_{0}\right|$ is square-integrable over the exterior of any disc about the origin in $R^{2}$. $U_{0}$ may be truncated to yield a function in $H_{1}\left(R^{2} ; C^{2}\right)$ which is useful for the present purposes. Let $\phi\left(x_{1}, x_{2}\right)$ be a real scalar-valued infinitely-differentiable function on $R^{2}$ which is identically equal to 1 outside the unit disc in $R^{2}$ and which vanishes in a neighborhood of the origin in $R^{2}$. Then the function $U_{1}=\phi U_{0}+(1-\phi)\left(\begin{array}{l}i \\ 0\end{array}\right)$ is in $H_{1}\left(R^{2} ; C^{2}\right)$ and has the following properties:

(i) $A_{0} U_{1}\left(x_{1}, x_{2}\right)=0$ when $x_{1}^{2}+x_{2}^{2} \geqq 1$.

(ii) $\left|U_{1}\left(x_{1}, x_{2}\right)\right|>0$ for all $\left(x_{1}, x_{2}\right)$.

Since, in particular, $U_{1}$ does not vanish on the closed unit disc in $R^{2}, A_{0}$ can be easily modified on the disc to yield an elliptic operator which annihilates $U_{1}$ : Denote $U_{1}$ and $A_{0} U_{1}$ by

$$
U_{1}=\left(\begin{array}{l}
u_{1} \\
u_{2}
\end{array}\right) \text { and } A_{0} U_{1}=\left(\begin{array}{l}
v_{1} \\
v_{2}
\end{array}\right)
$$

and put

$$
B\left(x_{1}, x_{2}\right)=\frac{-1}{\left|U_{1}\left(x_{1}, x_{2}\right)\right|^{2}}\left(\begin{array}{ll}
v_{1}\left(x_{1}, x_{2}\right) & 0 \\
v_{2}\left(x_{1}, x_{2}\right) & 0
\end{array}\right)\left(\begin{array}{rr}
u_{1}\left(x_{1}, x_{2}\right) & u_{2}\left(x_{1}, x_{2}\right) \\
-u_{2}\left(x_{1}, x_{2}\right) & u_{1}\left(x_{1}, x_{2}\right)
\end{array}\right) .
$$


Then the operator $A$ defined by

$$
A U\left(x_{1}, x_{2}\right)=A_{0} U\left(x_{1}, x_{2}\right)+B\left(x_{1}, x_{2}\right) U\left(x_{1}, x_{2}\right)
$$

is in $E\left(A_{0}, 1\right)$ and contains the nontrivial element $U_{1}$ of $H_{1}\left(R^{2} ; C^{2}\right)$ in its null-space.

It should be remarked that the easy construction of the above operator depends intimately on the fact that $U_{1}$ does not vanish on the closed unit disc in $R^{2}$. It is absolutely necessary, then, that $U_{1}$ take on complex values inside the unit disc. Finding a nonvanishing real-valued truncation of $U_{0}$ inside the unit disc is impossible for topological reasons.

4. Preparatory lemmas. Given a positive $R$ and an unperturbed elliptic operator

$$
A_{0} u(x)=\sum_{i=1}^{n} A_{i} \frac{\partial}{\partial x_{i}} u(x)
$$

on $H_{1}\left(\boldsymbol{R}^{n} ; \boldsymbol{C}^{k}\right)$ with constant coefficients and no zero-order term, consider the set

$$
M\left(A_{0}, R\right)=\left\{u \in H_{1}\left(R^{n} ; C^{k}\right): A_{0} u(x)=0 \text { if }|x| \geqq R\right\} .
$$

Certainly $N(A)$ is contained in $M\left(A_{0}, R\right)$ for every $A$ in $E\left(A_{0}, R\right)$. Put $A_{0}(\xi)=\sum_{i=1}^{n} A_{i} \xi_{i}$ for $\xi$ in $R^{n}$. The following is a fundamental estimate for the operator $A_{0}$.

Lemma 1. For $n \geqq 2$ and any positive $R$, there exists a positive constant $c$ for which $\|u\| \leqq c\left\|A_{0} u\right\|$ for every $u$ in $M\left(A_{0}, R\right)$. The constant $c$ may, in fact, be taken to be

$$
\left[\max _{|\xi|=1}\left|A_{0}(\xi)^{-1}\right|\right] \cdot\left[1+(2 \pi)^{-n}\left(\int_{|x| \leq 1}|x|^{-2} d x\right)\left(\int_{|x| \leqq R} d x\right)\right]^{1 / 2}
$$

when $n \geqq 3$, or

$$
\left[\max _{|\xi|=1}\left|A_{0}(\xi)^{-1}\right|\right] \cdot\left[1+(4 \pi)^{-1}\left(\int_{|x| \leqq R}|x|^{2} d x\right)\right]^{1 / 2}
$$

when $n=2$.

Proof. Suppose $u$ is in $M\left(A_{0}, R\right)$, i.e., suppose $A_{0} u=f$ has support in $\{x:|x| \leqq R\}$. Then, taking Fourier transforms, $i A_{0}(\xi) \hat{u}(\xi)=\hat{f}(\xi)$ and so, for nonzero $\xi$,

$$
|\xi(\xi)|^{2} \leqq\left|A_{0}(\xi)^{-1} \hat{f}(\xi)\right|^{2} \leqq c_{1}|\xi|^{-2}|\hat{f}(\xi)|^{2}
$$


where $c_{1}=\left[\max _{|\xi|-1}\left|A_{0}(\xi)^{-1}\right|\right]^{2}$. Then

$$
\begin{aligned}
\|u\|^{2} & =\|a\|^{2} \leqq \int_{R^{n}} c_{1}|\xi|^{-2}|\hat{f}(\xi)|^{2} d \xi \\
& =c_{1} \int_{|\xi| \geq 1}|\xi|^{-2}|\hat{f}(\xi)|^{2} d \xi+c_{2} \int_{|\xi| \leqq 1}|\xi|^{-2}|\hat{f}(\xi)|^{2} d \xi \\
& \leqq c_{1}\|\hat{f}\|^{2}+c_{2} \int_{|\xi| \leq 1}|\xi|^{-2}|\hat{f}(\xi)|^{2} d \xi .
\end{aligned}
$$

(Note that the remaining integral on the right-hand side will be finite for all $n \geqq 2$, since $|\xi|^{-2}|\hat{f}(\xi)|^{2}$ can be dominated by a constant multiple of the integrable function $|\hat{u}(\xi)|^{2}$.)

If $n \geqq 3$, then

$$
|\hat{f}(\xi)| \leqq(2 \pi)^{-n / 2} \int_{|x| \leq R}|f(x)| d x \leqq\left(c_{2}\right)^{1 / 2}\|f\|
$$

where $c_{2}=(2 \pi)^{-n} \int_{|x| \leq R} d x ;$ and so

$$
c_{1} \int_{|\xi| \leq 1}|\xi|^{-2}|\hat{f}(\xi)|^{2} d \xi \leqq c_{1} c_{2} c_{3}\|f\|^{2}
$$

where $c_{3}=\int_{|\xi| \leq 1}|\xi|^{-2} d \xi$. (Since $n \geqq 3, c_{3}$ is finite.) Then

$$
\|u\|^{2} \leqq c_{1}\|\hat{f}\|^{2}+c_{1} c_{2} c_{3}\|f\|^{2}=c_{1}\left(1+c_{2} c_{3}\right)\left\|A_{0} u\right\|^{2} .
$$

Taking the square root of both sides and substituting gives the desired inequality

$$
\begin{aligned}
\|u\| \leqq & {\left[\max _{|\xi|=1}\left|A_{0}(\xi)^{-1}\right|\right] } \\
& \cdot\left[1+(2 \pi)^{-n}\left(\int_{|x| \leqq 1}|x|^{-2} d x\right)\left(\int_{|x| \leq R} d x\right)\right]^{1 / 2}\left\|A_{0} u\right\| .
\end{aligned}
$$

If $n=2$, then $|\xi|^{-2}$ is not integrable over the unit disc, and a different approach must be taken. Since $f$ has compact support, $\hat{f}$ is continuously differentiable and, for $i=1,2$,

$$
\begin{aligned}
\left|\frac{\partial}{\partial \xi_{i}} \hat{f}(\xi)\right| & \leqq(2 \pi)^{-1} \int_{|x| \leq R}\left|x_{i}\right||f(x)| d x \\
& \leqq(2 \pi)^{-1}\left\{\int_{|x| \leq R}\left|x_{i}\right|^{2} d x\right\}^{1 / 2}\|f\| .
\end{aligned}
$$

Then for all $\xi$, 


$$
|\hat{f}(\xi)| \leqq|\hat{f}(0)|+|\xi|\left(c_{2}\right)^{1 / 2} \| f||
$$

where $c_{2}=(2 \pi)^{-2} \int_{|x| \leq R}|x|^{2} d x$.

Since $\int_{|\xi| \leq 1}|\xi|-2|\hat{f}(\xi)|^{2} d \xi$ is finite and $\hat{f}$ is continuous, it must be the case that $\hat{f}(0)=0$. Then one has $|\hat{f}(\xi)| \leqq|\xi|\left(c_{2}\right)^{1 / 2}\|f\|$ and so

$$
c_{1} \int_{|\xi| \leq 1}|\xi|^{-2}|\hat{f}(\xi)|^{2} d \xi \leqq c_{1} c_{2}\|f\|^{2}\left\{\int_{|x| \leq 1} d \xi\right\}=\pi c_{1} c_{2}\|f\|^{2} .
$$

Then

$$
\|u\|^{2} \leqq c_{1}\|\hat{f}\|^{2}+\pi c_{1} c_{2}\|f\|^{2}=c_{1}\left(1+\pi c_{2}\right)\left\|A_{0} u\right\|^{2} .
$$

Taking the square root of both sides and substituting gives the desired inequality

$$
\|u\| \leqq\left[\max _{|\xi|=1}\left|A_{0}(\xi)^{-1}\right|\right] \cdot\left[1+(4 \pi)^{-1}\left(\int_{|x| \leq R}|x|^{2} d x\right)\right]^{1 / 2}\left\|A_{0} u\right\| .
$$

This completes the proof.

When the independent variables are restricted to a compact set or manifold, one of the principal tools used in investigating the nullspaces of elliptic operators is the Rellich Compactness Theorem $[1$, p. 169]. Unfortunately, this famous result cannot be directly applied here, since the independent variables are allowed to range over all of $R^{n}$. However, it can be used to prove the following lemma, which provides a substitute adequate for the present purposes.

Lemma 2. For $n \geqq 2$ and any positive $R$, every subset of $M\left(A_{0}, R\right)$ which is bounded in $H_{1}\left(R^{n} ; C^{k}\right)$ is relatively compact in $L_{2}\left(R^{n} ; C^{k}\right)$.

PRoof. It will suffice to show that an arbitrary sequence $\left\{u_{m}\right\}$ in $M\left(A_{0}, R\right)$ which is bounded in $H_{1}\left(R^{n} ; C^{k}\right)$ contains a subsequence which is Cauchy in $L_{2}\left(R^{n} ; C^{k}\right)$. Given such a sequence, let $\phi$ be a scalar-valued infinitely-differentiable function on $R^{n}$ satisfying the following:

(i) $\phi(x)=1$ for $|x| \leqq R$.

(ii) $\phi(x)=0$ for $|x| \geqq 2 R$. Now

$$
\left\|\phi u_{m}\right\|_{1} \leqq \text { const }\left\|u_{m}\right\|_{1} \leqq \text { const }
$$

and the functions $\phi u_{m}$ have compact support, so it follows from the Rellich Compactness Theorem that there is a subsequence $\left\{\phi u_{m_{j}}\right\}$ which is Cauchy in $L_{2}\left(R^{n} ; C^{k}\right)$. The functions $(1-\phi) u_{m_{j}}$ do not necessarily comprise a Cauchy sequence in $L_{2}\left(R^{n} ; C^{k}\right)$, but one does have 


$$
A_{0}\left[(1-\phi) u_{m_{j}}\right]=\left[A_{0}(-\nabla \phi)\right] u_{m_{j}}+(1-\phi)\left[A_{0} u_{m_{j}}\right]=\left[A_{0}(-\nabla \phi)\right] u_{m_{j}}
$$

since $\left\{u_{m_{j}}\right\}$ is a subset of $M\left(A_{0}, R\right)$. It is immediately apparent, then, that the functions $A_{0}\left[(1-\phi) u_{m_{j}}\right]$ are bounded in $H_{1}\left(R^{n} ; C^{k}\right)$ and have support in $\{x:|x| \leqq 2 R\}$, and the Rellich Compactness Theorem implies that there is a subsequence $\left\{A_{0}\left[(1-\phi) u_{m_{j_{k}}}\right]\right\}$ which is Cauchy in $L_{2}\left(R^{n} ; C^{k}\right)$. But the functions $(1-\phi) u_{m_{j_{k}}}$ and their differences are in $M\left(A_{0}, 2 R\right)$, and so it follows from Lemma 1 that the sequence $\left\{(1-\phi) u_{m_{j_{k}}}\right\}$ is itself Cauchy in $L_{2}\left(R^{n} ; C^{k}\right)$. Finally, the sequence $\left\{u_{m_{j_{k}}}=\phi u_{m_{j_{k}}}+(1-\phi) u_{m_{j_{k}}}\right\}$ is a subsequence of $\left\{u_{m}\right\}$ which is Cauchy in $L_{2}\left(R^{n} ; C^{k}\right)$, and the proof is complete.

As usual, let $N(A)^{\perp}$ denote the orthogonal complement of $N(A)$ in $L_{2}\left(\boldsymbol{R}^{n} ; \boldsymbol{C}^{k}\right)$. The following lemma is a useful generalization of Lemma 1 .

Lemma 3. Let $A$ be an operator in $E\left(A_{0}, R\right)$ for some positive $R$. For $n \geqq 2$, there exists a positive constant $c$ for which $\|u\| \leqq c\|A u\|$ for every $u$ in $M\left(A_{0}, R\right) \cap N(A)^{\perp}$.

Proof. Suppose there exists no such estimate. Then there is a sequence $\left\{u_{m}\right\}$ contained in $M\left(A_{0}, R\right) \cap N(A)^{\perp}$ such that $\left\|u_{m}\right\|=1$ for all $m$ and $\left\|A u_{m}\right\|$ approaches zero as $m$ grows large. Applying the elliptic estimate for $A$, one obtains

$$
\left\|u_{m}\right\|_{1} \leqq c_{1}\left\|u_{m}\right\|+c_{2}\left\|A u_{m}\right\| \leqq \text { const }
$$

for all $m$. Then by Lemma 2 , the set $\left\{u_{m}\right\}$ is relatively compact and contains a subsequence $\left\{u_{m_{j}}\right\}$ which converges to an element $u_{0}$ of $L_{2}\left(R^{n} ; C^{k}\right)$. Since $A$ is a closed operator, $u_{0}$ is in $H_{1}\left(R^{n} ; C^{k}\right)$ and $A u_{0}=0$. Hence, $u_{0}$ is in $N(A)$. But $u_{0}$ is the limit of functions in $N(A)^{\perp}$, and so it must be the case that $u_{0}=0$. However, $\left\|u_{0}\right\|=$ $\lim _{j \rightarrow \infty}\left\|u_{m_{j}}\right\|=1$. This is a contradiction, and the lemma is proved.

5. Null-spaces of operators in $E\left(A_{0}, R\right)$. Let an unperturbed elliptic operator

$$
A_{0} u(x)=\sum_{i=1}^{n} A_{i} \frac{\partial}{\partial x_{i}} u(x)
$$

be given. The following theorem was proved by Lax and Phillips [3] in the case of an odd number of independent variables.

THEOREM 1. If $A$ is an operator in $E\left(A_{0}, R\right)$ for some positive $R$, then the dimension of $N(A)$ is finite for $n \geqq 2$.

Proof. $N(A)$ will be finite-dimensional if and only if $S$ 
$=\{u \in N(A):\|u\|=1\}$ is compact in $L_{2}\left(\boldsymbol{R}^{n} ; \boldsymbol{C}^{k}\right)$. Since $A$ is in $E\left(A_{0}, R\right)$, one has the elliptic estimate

$$
\|u\|_{1} \leqq c_{1}\|u\|+c_{2}\|A u\|
$$

for all $u$ in $H_{1}\left(R^{n} ; C^{k}\right)$. In particular, one has for every $u$ in $S$ that $\|u\|_{1} \leqq c_{1}$. Since $S$ is contained in $M\left(A_{0}, R\right)$, it follows from Lemma 2 that $S$ is relatively compact in $L_{2}\left(R^{n} ; C^{k}\right)$. Since $A$ is a closed operator, $S$ is a closed subset of $L_{2}\left(R^{n} ; C^{k}\right)$. Then $S$ must be compact in $L_{2}\left(\boldsymbol{R}^{n} ; \boldsymbol{C}^{k}\right)$, and the proof is complete.

Suppose $A$ and $A^{\prime}$ are two operators in $E\left(A_{0}, R\right)$. Then it follows from the elliptic estimate for such operators and the boundedness of the coefficients of the operator $\left(A-A^{\prime}\right)$ that there exist positive constants $c_{1}$ and $c_{2}$ for which

$$
\left\|\left(A-A^{\prime}\right) u\right\| \leqq c_{1}\|u\|+c_{2}\|A u\|
$$

for all $u$ in $H_{1}\left(\boldsymbol{R}^{n} ; \boldsymbol{C}^{k}\right)$. (Naturally, there is a similar estimate with different constants having $A^{\prime}$ on the right-hand side.) The constants $c_{1}$ and $c_{2}$ can be made arbitrarily small by taking the coefficients of $A^{\prime}$ sufficiently near those of $A$ uniformly in $R^{n}$. If one considers $A^{\prime}$ to be "near" $A$ if the constants $c_{1}$ and $c_{2}$ are "small," then this notion of nearness determines a topology on $E\left(A_{0}, \boldsymbol{R}\right)$. The following theorem shows that the dimension of $N(A)$ is an upper-semicontinuous function of the operators $A$ in $E\left(A_{0}, R\right)$ with this topology. (One cannot hope to have lower-semicontinuity of the dimension of the null-space as well. This is witnessed by the fact that the operator $A$ constructed in $\$ 2$, which has a nontrivial null-space, is homotopic in $E\left(A_{0}, 1\right)$ to its unperturbed operator $A_{0}$. Both upper- and lowersemicontinuity of the dimension of the null-space would, in this case, contradict the connectedness of the interval $[0,1]$.)

Theorem 2. Let $A$ and $A^{\prime}$ be operators in $E\left(A_{0}, R\right)$ for some positive $R$. For $n \geqq 2$, if $A^{\prime}$ is sufficiently near $A$ in the sense that the constants $c_{1}$ and $c_{2}$ are sufficiently small in the estimate

$$
\left\|\left(A-A^{\prime}\right) u\right\| \leqq c_{1}\|u\|+c_{2}\|A u\|
$$

for $u$ in $H_{1}\left(R^{n} ; C^{k}\right)$, then the dimension of $N\left(A^{\prime}\right)$ is no greater than the dimension of $N(A)$.

Proof. Suppose that $c_{2}$ is less than 1 and that together $c_{1}$ and $c_{2}$ are sufficiently small that $c_{1} /\left(1-c_{2}\right)<c^{-1}$ where $c$ is the constant in the estimate of Lemma 3 for this choice of $R$ and $A$. For all $u$ in $H_{1}\left(R^{n} ; C^{k}\right)$, one has 


$$
c_{1}\|u\|+c_{2}\|A u\|+\left\|A^{\prime} u\right\| \geqq\left\|A^{\prime} u\right\|+\left\|\left(A-A^{\prime}\right) u\right\| \geqq\|A u\|
$$

or

$$
\left\|A^{\prime} u\right\| \geqq\left(1-c_{2}\right)\|A u\|-c_{1}\|u\| .
$$

Now $\left(1-c_{2}\right)$ is positive, and so one can apply Lemma 3 to obtain, for all $u$ in $M\left(A_{0}, R\right) \cap N(A)^{\perp}$,

$$
\left\|A^{\prime} u\right\| \geqq\left[\left(1-c_{2}\right) c^{-1}-c_{1}\right]\|u\|=c^{\prime}\|u\| .
$$

Since, by assumption, $c_{1} /\left(1-c_{2}\right)$ is less than $c^{-1}$, the constant $c^{\prime}$ $=\left[\left(1-c_{2}\right) c^{-1}-c_{1}\right]$ is positive.

Since $N\left(A^{\prime}\right)$ is a subset of $M\left(A_{0}, R\right)$, one can see from the estimate $\left\|A^{\prime} u\right\| \geqq c^{\prime}\|u\|$ on $M\left(A_{0}, R\right) \cap N(A)^{\perp}$ that $N\left(A^{\prime}\right) \cap N(A)^{\perp}=\{0\}$. It follows that the dimension of $N\left(A^{\prime}\right)$ is no greater than the dimension of $N(A)$; otherwise, there would necessarily be a nonzero element of $N\left(A^{\prime}\right) \cap N(A)^{\perp}$. This completes the proof.

Note that Theorem 2 implies, in particular, that if an operator $A$ in $E\left(A_{0}, R\right)$ is sufficiently near the unperturbed operator $A_{0}$ in the sense that the constants $c_{1}$ and $c_{2}$ are sufficiently small in the estimate

$$
\left\|\left(A-A_{0}\right) u\right\| \leqq c_{1}\|u\|+c_{2}\left\|A_{0} u\right\|
$$

for $u$ in $H_{1}\left(\boldsymbol{R}^{n} ; \boldsymbol{C}^{k}\right)$, then $N(A)=\{0\}$. It should be remarked, however, that this result can be obtained in a decidedly superior manner: Merely repeat the first paragraph of the proof of Theorem 2, substituting the constant $c$ in the estimate of Lemma 1 for the constant $c$ in the estimate of Lemma 3, to obtain a positive constant $c^{\prime}$ for which the estimate $\|A u\| \geqq c^{\prime}\|u\|$ holds for all $u$ in $M\left(A_{0}, R\right)$. The result follows immediately, and one gains by eliminating Lemmas 2 and 3 and by using a directly calculable constant in place of a constant which can only be asserted to exist via an indirect argument.

\section{REFERENCES}

1. L. Bers, F. John and M. Schecter, Partial differential equations, Lectures in Appl. Math., vol. 3, Interscience, New York, 1964. MR 29 \#346.

2. T. Kato, Perturbation theory for linear operators, Die Grundlehren der math. Wissenschaften, Band 132, Springer-Verlag, New York, 1966. MR 34 \#3324.

3. P. D. Lax and R. S. Phillips, Lectures on scattering theory, Summer Institute on Scattering Theory at Flagstaff, Arizona, 1969; see also Scattering theory, Rocky Mt. J. Math. 1 (1971), 173-223.

4. P. D. Lax and R. S. Phillips, Scattering theory, Pure and Appl. Math., vol. 26, Academic Press, New York, 1967. MR 36 \#530.

Texas Tech University, Lubbock, Texas 79409 